\title{
Towards Ontological Blending
}

\author{
Joana Hois, Oliver Kutz, Till Mossakowski, and John Bateman \\ SFB/TR 8 Spatial Cognition, University of Bremen, Germany \\ \{joana, okutz, till\}@informatik. uni-bremen.de, \\ batemaneuni-bremen. de
}

\begin{abstract}
We propose ontological blending as a new method for 'creatively' combining ontologies.
\end{abstract}

In contrast to other combination techniques that aim at integrating or assimilating categories and relations of thematically closely related ontologies, blending aims at 'creatively' generating new categories and ontological definitions on the basis of input ontologies whose domains are thematically distinct but whose specifications share structural or logical properties. As a result, ontological blending can generate new ontologies and concepts and allows a more flexible technique for ontology combination than existing methods. The approach is inspired by conceptual blending in cognitive science, and draws on methods from ontological engineering, algebraic specification, and computational creativity in general.

Well-known techniques directed towards unifying the semantic content of different ontologies, namely techniques based on matching, aligning, or connecting ontologies, are ill-suited for generating new conceptual schemas from existing ontologies as suggested by the general methodology of conceptual blending introduced by Fauconnier and Turner [3]: here, the blending of two thematically rather different conceptual spaces yields a new conceptual space with emergent structure, selectively combining parts of the given spaces whilst respecting common structural properties. A classic example for this is the blending of the theories of house and boat yielding as blends the theories of houseboat and boathouse, but also the blended theory of amphibious vehicle [6].

Conceptual blending inspires a structural and logic-based approach to 'creative' ontological engineering which allows the creation of new ontologies with emergent structure. Ontologies developed this way can be used, e.g., for applications in the area of computational creativity or analyses of artistic processes [2]. We believe that the principles governing ontological blending are quite distinct from the rather loose principles employed in blending phenomena in language or poetry, or the rather strict principles ruling blending in mathematics.

Our approach to ontological blending follows a line of research in which blending processes are primarily controlled through mappings and their properties [5, 4, 12]. By introducing blending to ontology languages, we propose a technique to combine two thematically different ontologies to create the blendoid, an ontology describing a newly created domain. The blendoid creatively mixes information from input ontologies on the basis of their structural commonalities and combines their axiomatisation, raising the following challenges: (1) when combining the terminologies of two ontologies, the 
shared semantic structure is of particular importance to steer possible combinations; this shared semantic structure leads to the notion of base ontology and the problem of computing it. (2) Having established a shared semantic structure, there is typically still a huge number of blending possibilities: here, optimality principles for selecting blends take on a central role. We approach these challenges as follows: we

- differentiate alignment, matching, analogical reasoning, and conceptual blending, vis-à-vis ontological blending;

- give an abstract definition of ontological blendoids capturing the basic intuitions of conceptual blending in the ontological setting;

- provide a structured approach to ontology languages, in particular to OWL-DL, by defining the language hOWL. This combines the simplicity and good tool support for OWL with the more complex blending facilities of OBJ3 [7] or Haskell [8];

The tool HeTs, the HETCASL [11] language, and in particular hOWL, provide an ideal starting point for developing the algorithmic side of the theory further. They (1) support various ontology language and their heterogeneous integration, and allow the specification of theory interpretations and other morphisms between ontologies [9]; (2) support the computation of colimits as well as the approximation of colimits in the heterogeneous case [1]; (3) provide (first) solutions for automatically computing a base ontology through ontology intersection [10].

These issues constitute almost completely new research questions in ontology research.

\section{References}

1. Codescu, M., Mossakowski, T.: Heterogeneous colimits. In: Proc. of MoVaH-08 (2008)

2. Colton, S.: Towards ontology use, re-use and abuse in a computational creativity collective. In: Kutz, O., Hois, J., Bao, J., Grau, B.C. (eds.) Modular Ontologies - Proceedings of the Fourth International Workshop (WoMO'2010). pp. 1-4. IOS Press (2010)

3. Fauconnier, G., Turner, M.: The Way We Think: Conceptual Blending and the Mind's Hidden Complexities. Basic Books (2003)

4. Forbus, K., Falkenhainer, B., Gentner, D.: The structure-mapping engine. Artificial Intelligence 41, 1-63 (1989)

5. Gentner, D.: Structure mapping: A theoretical framework for analogy. Cognitive Science 7(2), 155-170 (1983)

6. Goguen, J.A., Harrell, D.F.: Style: A Computational and Conceptual Blending-Based Approach. In: The Structure of Style: Algorithmic Approaches to Understanding Manner and Meaning. Springer (2009)

7. Goguen, J.A., Malcolm, G.: Algebraic Semantics of Imperative Programs. MIT (1996)

8. Kuhn, W.: Modeling the Semantics of Geographic Categories through Conceptual Integration. In: Proc. of GIScience 2002. pp. 108-118. Springer (2002)

9. Kutz, O., Lücke, D., Mossakowski, T.: Heterogeneously Structured Ontologies-Integration, Connection, and Refinement. In: Proc. KROW 2008. CRPIT, vol. 90, pp. 41-50. ACS (2008)

10. Kutz, O., Normann, I.: Context Discovery via Theory Interpretation. In: Workshop on Automated Reasoning about Context and Ontology Evolution, ARCOE-09 (IJCAI-09) (2009)

11. Mossakowski, T., Maeder, C., Lüttich, K.: The Heterogeneous Tool Set. In: TACAS. LNCS, vol. 4424, pp. 519-522. Springer (2007)

12. Pereira, F.C.: Creativity and Artificial Intelligence: A Conceptual Blending Approach, Applications of Cognitive Linguistics (ACL), vol. 4. Mouton de Gruyter (2007) 